\title{
Multi-wavelength analysis of high energy electrons in solar flares: a case study of August 20, 2002 flare
}

\author{
Jana Kašparová and Marian Karlický \\ Astronomical Institute, Academy of Sciences of the Czech Republic, \\ 25165 Ondřejov, Czech Republic \\ Eduard P. Kontar \\ Department of Physics and Astronomy, University of Glasgow, \\ Glasgow G12 8QQ, UK \\ Richard A. Schwartz \\ Science Systems 86 Applications, Inc. at NASA Goddard Space Flight Center, \\ Greenbelt, MD 20771, USA \\ Brian R. Dennis \\ NASA Goddard Space Flight Center, Greenbelt, MD 20771, USA
}

\begin{abstract}
A multi-wavelength spatial and temporal analysis of solar high energy electrons is conducted using the August 20, 2002 flare of an unusually flat $\left(\gamma_{1}=1.8\right)$ hard X-ray spectrum. The flare is studied using RHESSI, H $\alpha$, radio, TRACE, and MDI observations with advanced methods and techniques never previously applied in the solar flare context. A new method to account for X-ray Compton backscattering in the photosphere (photospheric albedo) has been used to deduce the primary $\mathrm{X}$-ray flare spectra. The mean electron flux distribution has been analysed using both forward fitting and model independent inversion methods of spectral analysis. We show that the contribution of the photospheric albedo to the photon spectrum modifies the calculated mean electron flux distribution, mainly at energies below $\sim 100 \mathrm{keV}$. The positions of the $\mathrm{H} \alpha$ emission and hard $\mathrm{X}$-ray sources with respect to the current-free extrapolation of the MDI photospheric magnetic field and the characteristics of the radio emission provide evidence of the closed geometry of the magnetic field structure and the flare process in low altitude magnetic loops. In agreement with the predictions of some solar flare models, the hard X-ray sources are located on the external edges of the $\mathrm{H} \alpha$ emission and show chromospheric plasma heated by the non-thermal electrons. The fast changes of $\mathrm{H} \alpha$ intensities are located not only inside the hard X-ray sources, as expected if they are the signatures of the chromospheric response to the electron bombardment, but also away from them.
\end{abstract}

Keywords: solar flares, RHESSI, $\mathrm{H} \alpha$, radio, albedo corrections

\section{Introduction}

Analyses of the impulsive phase of solar flares detected in various electromagnetic wavelength ranges are important for understanding the acceleration and propagation of particles. The accelerated electrons reveal their presence e.g. in hard X-ray and radio emissions, and cause

(c) 2018 Kluwer Academic Publishers. Printed in the Netherlands. 
rapid heating of the chromosphere and subsequent emission in the hydrogen $\mathrm{H} \alpha$ line.

One of the key questions is the form of the accelerated electron distributions. Their determination from the hard X-ray spectra depends not only on the emission mechanism but also on the processes affecting the propagation of the particles. As suggested by Brown, Emslie, and Kontar (2003), it is the mean electron flux distribution $\bar{F}(E)$ depending only on the bremsstrahlung cross section that should be used as a reference for both observational hard X-ray spectral analyses and theoretical models of electron acceleration and propagation. The mean electron flux distribution can be determined either by inverting the photon spectra (Johns and Lin, 1992; Piana et al., 2003) or by a forward fitting method usually assuming a power-law form of $\bar{F}(E)$ (Holman et al., 2003). Observed hard X-ray spectra also contain a contribution of photons backscattered in the photosphere and this will modify the calculated $\bar{F}(E)$ (Bai and Ramaty, 1978; Alexander and Brown, 2002).

Besides the X-ray emission, numerous types of radio emission in the metric and decimetric range are usually observed during flares. They provide further information about the acceleration and propagation of the electron beams and plasma parameters in the emission region (Karlický, 1997).

According to standard solar flare models (e.g. Dennis and Schwartz, 1989), the accelerated electrons provide one of the mechanisms for the flare energy transport from the release site to the lower atmospheric layers. As the accelerated electrons propagate along the magnetic field lines toward the photosphere, they lose their kinetic energy mainly via Coulomb collisions (Brown, 1971) in the lower corona and chromospheric layers. The chromospheric response to the beam energy deposition determines the characteristics of optical and UV emission from the flare loops. Numerical models of chromospheric response to pulsed electron beam heating (e.g. Canfield and Gayley, 1987; Heinzel, 1991) predict the time correlation of hard X-ray and $\mathrm{H} \alpha$ emission recently analysed e.g. by Trottet et al. (2000) and Wang et al. (2000). Their results show that hard X-rays and $\mathrm{H} \alpha$ intensities in some flare kernels exhibit time correlations in the time range from subseconds to $\sim 10 \mathrm{~s}$.

Some flare models (e.g. Heyvaerts, Priest, and Rust, 1977; Cargill and Priest, 1983) and observations (Czaykowska et al., 1999) suggest that $\mathrm{H} \alpha$ kernels are located between the upflows of the beam heated plasma and the downflows of the cool plasma in the loops disconnected from the reconnection site. The leading edges of the $\mathrm{H} \alpha$ emission close to the newly reconnected loops are supposed to be also heated by the accelerated particles, which are detectable e.g. as the hard X-ray 
sources. Comparison of the spatial distribution of Yohkoh hard X-ray sources and $\mathrm{H} \alpha$ flare kernels was done by e.g. Asai et al. (2002). They found that many $\mathrm{H} \alpha$ kernels brighten successively during the evolution of the flare ribbons, but only a few radiation sources were seen in the hard X-ray images. They suggest that this discrepancy may be the result of the low dynamic range capability of the Yohkoh Hard X-ray Telescope.

In this paper, we analyse the impulsive phase of the August 20, 2002 solar flare, which is characterised by a very flat photon spectrum at the X-ray burst maximum (spectral index $\gamma_{1}=1.8$ ). Similar flat spectra have been reported, e.g. by Nitta, Dennis, and Kiplinger (1990) and Fárník, Hudson, and Watanabe (1997). The event presented here is the first such flat spectrum spectrum observed with the high energy resolution of the Reuven Ramaty High Energy Solar Spectroscopic Imager (RHESSI) (Lin et al., 2002). We show the influence of photons backscattered in the photosphere on the determination of the mean electron flux distribution and compare results obtained by inversion and forward fitting methods. We also study the spatial correlation of $\mathrm{H} \alpha$ emission and its time changes with hard X-ray sources. In section 2 we describe the global behaviour of the event with regard to hard $\mathrm{X}$-ray and radio fluxes. Sections 3 and 4 present the RHESSI, $\mathrm{H} \alpha$ and magnetic field observations, methods, and results. Finally, the results are discussed in section 5 and the conclusions are given in section 6 .

\section{Global event description}

The analysed flare is well suited for a multi-wavelength study of the electron propagation because its observations provide a set of simultaneous data of comparable time and spatial resolution in the wavelengths directly related to the accelerated particles: RHESSI hard X-ray spectra and images, $\mathrm{H} \alpha$ images (Kanzelhöhe observatory), and radio and microwave fluxes (Bern, Ondřejov, and Zürich facilities).

The flare was detected by GOES satellites on August 20, 2002. It started at 08:25 UT and reached its maximum at 08:26 UT as an M3.4 flare. The 1B $\mathrm{H} \alpha$ flare was reported in NOAA AR 0069 at S10W38, starting at 08:25 UT, peaking at 08:26 UT, and ending at 08:37 $\mathrm{UT}^{1}$.

A global overview of the flare evolution in $\mathrm{dm} / \mathrm{m}$ radio waves and RHESSI X-rays is shown in Figure 1. The RHESSI flare was first detected at 08:24:32 UT with the increase in flux in the 12 - $25 \mathrm{keV}$ energy band. Then, with some time delays (at $~ 08: 24: 45 \mathrm{UT}$ ), bursts in higher

\footnotetext{
${ }^{1}$ http://www.sec.noaa.gov/weekly/pdf2002/prf1408.pdf
} 


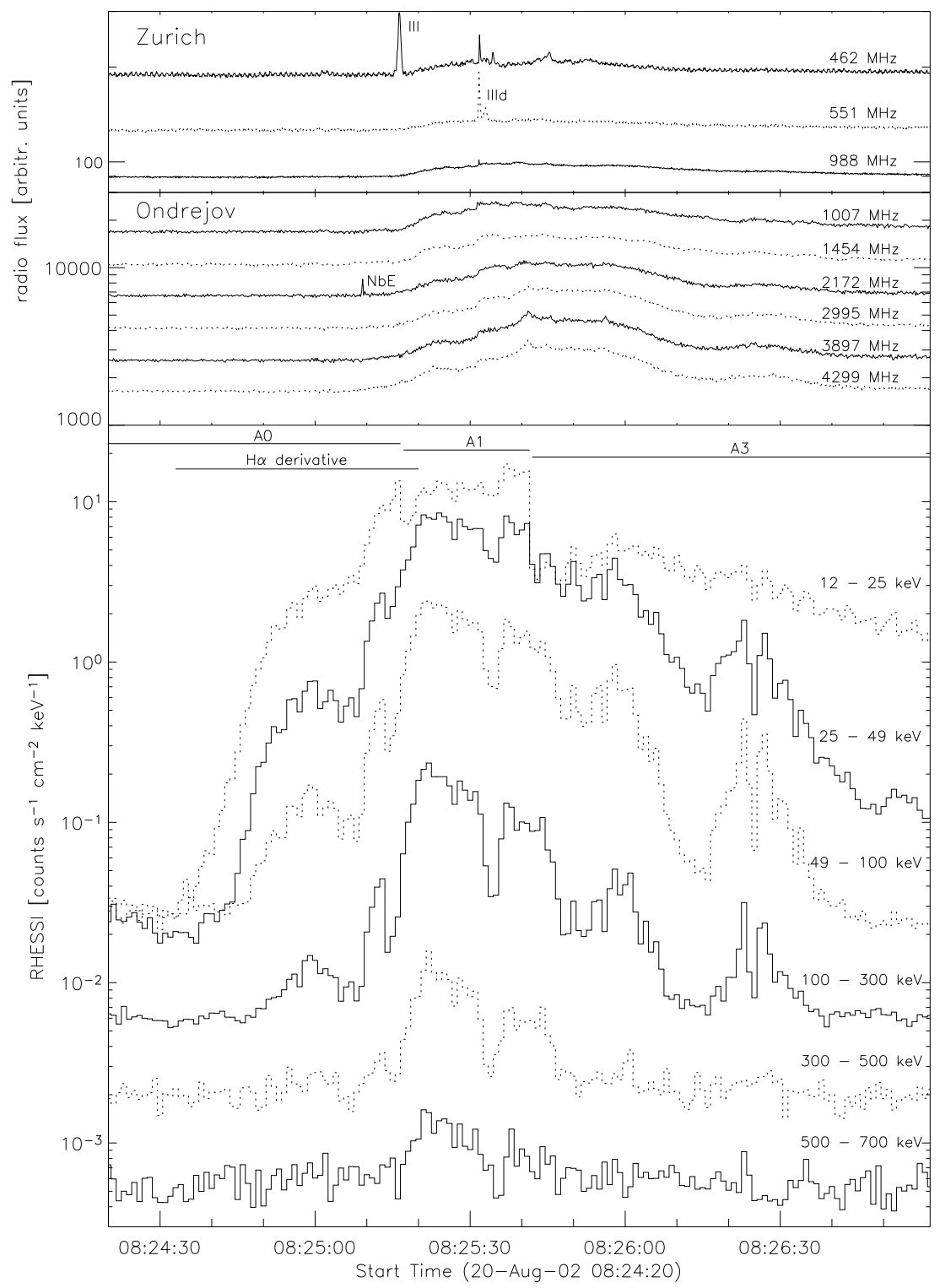

Figure 1. Time evolution of radio and hard X-ray fluxes during the August 20, 2002 flare from observations made with Zürich and Ondřejov radiospectrometers and RHESSI. Narrowband emission (NbE), type III, and IIId bursts are annotated on the Ondřejov and Zürich data plots. For display purposes the RHESSI count flux at the energy band $300-500 \mathrm{keV}$ was multiplied by a factor of 2, radio fluxes were shifted with a step of $\log \Delta=0.2$ from the fluxes at $4299 \mathrm{MHz}$ (Ondřejov) and $988 \mathrm{MHz}$ (Zürich). Attenuators states A0, A1, and A3 and the time interval in which the derivative of $\mathrm{H} \alpha$ intensity was analysed are indicated by the lines at the top of the RHESSI data plot. The abrupt changes in RHESSI flux in the $12-25 \mathrm{keV}$ energy band at $\sim 08: 25: 17$ UT and $\sim 08: 25: 41$ UT are caused by the attenuator changes $(\mathrm{A} 0 \rightarrow \mathrm{A} 1$ and $\mathrm{A} 1 \rightarrow \mathrm{A} 3$, respectively) at these times. 
and higher energy bands followed. During the starting phase of the Xray emission at 08:24:30 - 08:25:08 UT, no radio bursts were observed in the $\mathrm{dm} / \mathrm{m}$ range. The first weak and narrowband emission $(\mathrm{NbE})$ in the dm-range was registered at 08:25:08 UT in the $2000-2200 \mathrm{MHz}$ frequency range, followed by the type III burst below $500 \mathrm{MHz}$ at 08:25:16 UT.

The most energetic part of this flare occurred during the time interval 08:25:15 - 08:25:50 UT, when significant hard X-ray emission was detected up to $7 \mathrm{MeV}$ (see also data from the SONG instrument on board CORONAS-F; Myagkova, priv. comm.) and microwave emission up to $89.4 \mathrm{GHz}$. The turn-over frequency between optically thick and thin part of the radio emission was shifted to values between 19.6 and $50 \mathrm{GHz}$. At that time the radio emission in the dm-range also reached the maximum and a very fast drifting $\left(\sim-4000 \mathrm{MHz} \mathrm{s}{ }^{-1}\right)$ and short-lasting $(\sim 0.1 \mathrm{~s})$ type IIId burst (relativistic type III burst - see Poquerusse, 1994) was observed in the $400-1000 \mathrm{MHz}$ range at 08:25:31.8 UT. In the decimetric range $(2000-4500 \mathrm{MHz})$ this phase was characterised by broadband pulsations.

\section{RHESSI data analysis}

RHESSI X-ray data were analysed with the RHESSI Data Analysis Software (Hurford et al., 2002; Schwartz et al., 2002). During the flare, different attenuators were automatically placed in front of all detectors to absorb soft X-rays and reduce pulse pile-up. The attenuator states $\mathrm{A} 0, \mathrm{~A} 1$, and $\mathrm{A} 3$ (no attenuator, thin attenuator, and both thick and thin attenuator, respectively) are indicated on Figure 1. Corrections for the effects of the different attenuator states are available for all times except when the attenuators are moving, a period of $\sim 1 \mathrm{~s}$.

\subsection{IMAGING}

Image reconstruction was performed with both the CLEAN and the PIXON algorithms using data from the front segments of the detectors. Detector 7 was used only for the images in the energy bands above $7 \mathrm{keV}$ due to its energy threshold of about $7 \mathrm{keV}$. Detector 1 and 2 mainly added noise to the images indicating that there was no significant source structure with an angular scale finer than $\sim 4$ arcsecond. Therefore, these detectors were excluded.

The CLEAN algorithm was used for obtaining morphology and time evolution of X-ray sources, see sections 4.1 and 4.3, Figures 5, 6, and 9. Since there was no source component as large as or larger than FWHM 
of the detector 8 (107 arcsec), the imaging fields of view in all CLEAN images were set smaller than this FWHM and consequently detectors 8 and 9 were also not used in the CLEAN image reconstruction.

The PIXON algorithm (Puetter and Piña 1994; Metcalf et al., 1996) is known to suppress spurious sources and to have high photometric accuracy (Metcalf et al., 1996; Alexander and Metcalf, 1997). We have used the PIXON imaging technique for imaging spectroscopy at the burst maximum, see section 3.3.

\subsection{Spectroscopy}

We analysed data summed over the eight front segments. Detector 2 was excluded since its energy threshold is about $20 \mathrm{keV}$ and its energy resolution is about $10 \mathrm{keV}$. The data were corrected for pulse pile-up and decimation. Full 2D detector response matrices, each corresponding to the applied attenuator state, were used to convert input photon fluxes to count rates. The response matrix accounts for the efficiency and resolution of the detectors, the absorption by the attenuators, grids, and all other material above the detectors, and all other known instrumental effects (Smith et al., 2002).

The time bin for RHESSI spectra was $2 \mathrm{~s}$ ( $\approx$ a half rotation), which ensures that the rapid modulation produced by the grids does not have any distorting effect on the spectra. The RHESSI spectra were analysed in the time interval 08:25:10 - 08:26:00 UT and were fitted from $8 \mathrm{keV}$ up to typically $400 \mathrm{keV}$, depending on the signal-to-noise ratio.

RHESSI data of this flare were contaminated by an electron precipitation $^{2}$ starting at 08:16 UT and detected up to $300 \mathrm{keV}$. Therefore, a special care was taken to estimate the background due to the electron precipitation in the $12-300 \mathrm{keV}$ energy range while standard techniques were used at other energies. In the 12 - $40 \mathrm{keV}$ energy range, the background was estimated using count rates from the onboard charged particle detector whose lower threshold $(50 \mathrm{keV})$ is generally triggered during electron precipitation events (Smith et al., 2002). Analysing several precipitation events which occured on other orbits two days before and after the flare, we have found a good correlation between the particle count rate and the front count rate in this energy range. Therefore, count rates from the particle detector can serve as a proxy for the bremsstrahlung of the precipitating electrons as detected by germanium detectors in this energy band.

At higher energies the germanium detector count rates during the precipitation event are generally dominated by the bremsstrahlung that

\footnotetext{
2 hsi_20020820_071620_rate.png and hsi_20020820_071620_part_rate.png at http://hessi.ssl.berkeley.edu/hessidata/metadata/2002/08/20/
} 
originates from the electrons interacting in the Earth's atmosphere and generally does not follow the time history of the particle count rate (Smith, priv. comm.). Having taken this into account, the precipitation component in the $40-300 \mathrm{keV}$ energy range was estimated with a cubic fit to the shoulders of the precipitation event detected before and after the flare.

The uncertainty of the estimated precipitation component in the $12-300 \mathrm{keV}$ energy range was estimated to be $\leq 50 \%$. Despite this high value, any residual precipitation component in the background subtracted count spectra in the $12-300 \mathrm{keV}$ energy range is $\leq 10 \%$ at the edges of the analysed time interval and decreases to several percent with the increasing flare flux. Thus, the precipitation event does not affect our results in the analysed time and energy range.

\subsection{Flat Photon SPECTRUm at the Flare maximum}

In order to evaluate the power-law indices of the photon spectra and to make comparisons with previous works, we adopted a standard photon spectrum model $I$ consisting of a double power-law non-thermal component $I_{\text {nt }}$ (e.g. Lin and Schwartz, 1987) and a Maxwellian isothermal component $I_{\mathrm{th}}: I=I_{\mathrm{th}}+I_{\mathrm{nt}}$.

$$
I_{\mathrm{nt}}(\epsilon)=\left\{\begin{array}{ll}
a \epsilon^{-\gamma_{1}} & \epsilon<\epsilon_{\mathrm{b}} \\
a \epsilon_{\mathrm{b}}^{-\gamma_{1}+\gamma_{2}} \epsilon^{-\gamma_{2}} & \epsilon \geq \epsilon_{\mathrm{b}}
\end{array},\right.
$$

where $\epsilon, \epsilon_{\mathrm{b}}, \gamma_{1}, \gamma_{2}$, and $a$ are the photon energy, the break energy in $\mathrm{keV}$, the power-law index below and above $\epsilon_{\mathrm{b}}$, and $a$ is for normalisation, respectively. We used the forward fitting method and fitted the background subtracted X-ray spectra with the model spectrum $I$. The model spectrum was convolved with the full $2 \mathrm{D}$ detector response matrix to compute the model count spectrum. Parameters of the best

fit model were found by searching for a minimum $\chi_{\min }^{2}$ between the measured and the model count rate spectrum.

The lowest value of the power-law index $\gamma_{1}$ was found at the burst maximum as seen in $>25 \mathrm{keV}$ X-rays, at 08:25:22 UT, when it dropped to 1.8. Note that this value lies outside of the range of common values of photon power-law index, $\langle 2,7\rangle$, as reported e.g. by Dennis (1985). The flatness of the spectrum does not result from the contamination by a generally flat spectrum of the precipitation event because the residual precipitation component in the background-subtracted count spectrum was estimated to be $\leq 1 \%$ in the whole energy range at 08:25:22 UT. Hence, we believe that the flat spectrum is of solar origin.

This conclusion is further supported by the results from the imaging spectroscopy using the PIXON imaging method (Hurford et al., 2002). 
RHESSI PIXON images are constructed by finding the simplest model which is consistent with the counts observed during the time interval. The model used included a source image and a component that accounts for the counts unmodulated by the grids. This additional component prevents the unmodulated counts from being included in the source image. Thus, the PIXON image is not contaminated by this background and the PIXON image reconstruction provides an independent method of determining the spatially integrated flare spectrum. We reconstructed the PIXON images in several energy bands for $4.141 \mathrm{~s}$ $(\sim 1$ spin period) centred at the burst maximum (08:25:22 UT) and determined a PIXON photon spectrum from the total photon fluxes above the $10 \%$ contour level of the corresponding PIXON image. Since the uncertainty in the spectra from the image reconstruction is $\sim 10 \%$ (Hurford, priv. comm.), and the PIXON photon spectrum agrees within uncertainties of the same order with the flat photon spectrum up to $300 \mathrm{keV}$ (i.e. the highest energy for which the sources were detectable), we may conclude that the background subtraction procedure, see section 3.2, resulted in a solar count spectrum not significantly distorted by the precipitation component.

\subsection{Correction For PHOtospheric AlBedo AND ITS IMPLICATIONS ON MEAN ELECTRON FLUX DISTRIBUTION}

Primary photons with energies larger than $15 \mathrm{keV}$ emitted downwards have a high probability of being reflected due to Compton backscattering in the photosphere. These albedo photons then modify both the spectrum and the intensity of the observed hard X-rays. According to Bai and Ramaty (1978), the reflectivity of a power-law photon spectrum from the isotropic source, which is assumed here, is a function of the photon energy and significantly depends on the photon spectral index $\gamma$. The reflectivity peaks at $\sim 35 \mathrm{keV}$ and increases as $\gamma$ decreases. The total photon spectrum, that is the primary one plus the component composed of the reflected photons, is then characterised by a lower $\gamma$ than that of the primary spectrum. For a spectral index $\gamma \sim 1.8$, the reflected photons at $35 \mathrm{keV}$ can be as high as $80 \%$ of the primary flux. The amount of backscattered photons generally depends on the anisotropy of the primary hard X-ray source and the pitch-angle distribution of the beam electrons. But, the dependency is rather weak due to strong angular smoothing effect of the bremsstrahlung cross section. A detailed study of beam electron distributions is out of scope of this paper. However, the assumption of isotropy of the hard X-ray source provides a lower limit for the photospheric albedo correction. 
For this flare we studied the influence of the photospheric albedo correction on the mean electron flux distribution $\bar{F}(E)$. We used two approaches. First, $\bar{F}(E)$ was approximated by a double power-law function

$$
\bar{F}(E)=\left\{\begin{array}{ll}
A E^{-\delta_{1}} & E_{\text {low }} \leq E<E_{\text {break }} \\
A E_{\text {break }}^{-\delta_{1}+\delta_{2}} E^{-\delta_{2}} & E_{\text {break }} \leq E \leq E_{\text {high }}
\end{array},\right.
$$

where $E, E_{\text {low }}, E_{\text {break }}, E_{\text {high }}, \delta_{1}, \delta_{2}$, and $A$ are the electron energy, the low-energy cutoff, the break energy, the high-energy cutoff in keV, the power-law index below and above $E_{\text {break }}$, and normalisation, respectively. The mean electron flux distribution represents the density weighted electron flux distribution in the source, and its determination is equivalent to the determination of the electron flux distribution assuming thin-target bremsstrahlung $I_{\text {thin }}(\epsilon)$ (Brown, Emslie and Kontar, 2003)

$$
I_{\text {thin }}(\epsilon)=\frac{\bar{n} V}{4 \pi R^{2}} \int_{\epsilon}^{E_{\text {high }}} \bar{F}(E) Q(\epsilon, E) \mathrm{d} E,
$$

where $\bar{n}$ is the mean intensity in the source volume $V, R=1 \mathrm{AU}$ is the distance of the emitting source, and $Q(\epsilon, E)$ is the isotropic cross section for bremsstrahlung (Haug, 1997). Using the forward fitting method described in section 3.3, the background subtracted photon spectra were fitted with a Maxwellian isothermal component $I_{\text {th }}$ plus the thintarget bremsstrahlung $I_{\text {thin }}$ of the electron flux model $\bar{F}(E)$ and the best-fit values of thermal plasma and electron flux parameters (the fit provides $\bar{n} V \bar{F}(E))$ were obtained. $E_{\text {high }}$ was kept fixed at $7 \mathrm{MeV}$ since setting the parameter free did not significantly improve the fits.

Second, the mean electron flux distribution function was derived by a regularised inversion procedure (Piana et al., 2003; Kontar et al., 2004, 2005a) using the photon spectra (determined with the forward fitting method) as input. Electron energies were determined in the energy range typically 10 - $400 \mathrm{keV}$, the upper energy depending on the signalto-noise ratio.

\subsubsection{Photospheric albedo}

Background subtracted photon spectra were corrected for photospheric albedo using the method of an angle dependent Green's function for Xray Compton backscattering (Kontar et al., 2005b), where the Green's function represents the probability density of backscattering of a photon of initial energy $\epsilon_{0}$ into the observer's direction with energy $\epsilon$. This approach is independent of the spectral characteristics of the primary photon spectra and allows any form of the primary photon spectra to be deduced, not just a power-law spectrum as was done e.g. by Bai and 


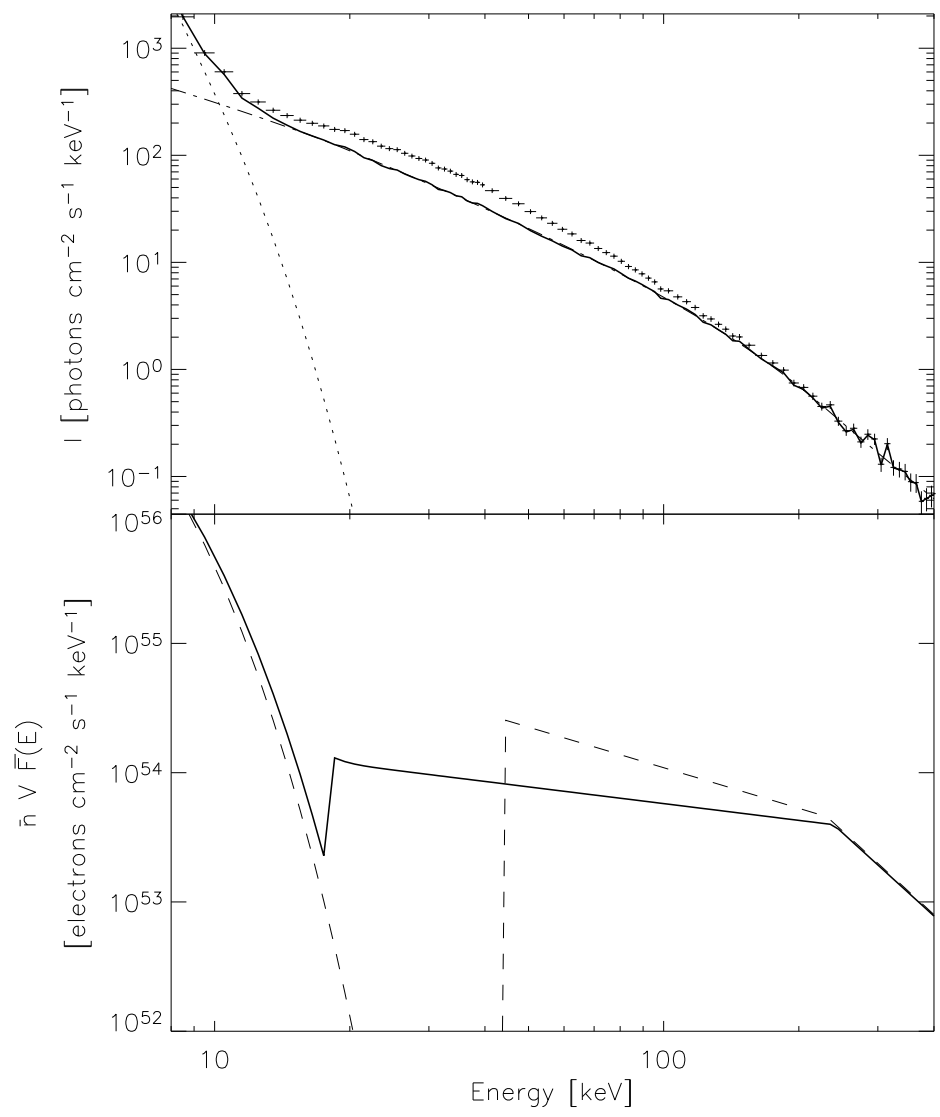

Figure 2. Influence of the photospheric albedo on the photon and mean electron flux spectrum at 08:25:22 UT (intergration time 2s). Top panel shows the total (crosses) and primary photon spectrum (-). Horizontal sizes of crosses correspond to widths of energy bands, vertical sizes represent $\pm 1 \sigma$ statistical plus systematic uncertainties. Best fit to the primary photon flux is composed of the isothermal $I_{\text {th }}(\cdots)$ and thin-target $I_{\text {thin }}(-\cdot-)$ component. Bottom panel compares forward fitted $\bar{n} V \bar{F}(E)$ derived from the total (- - ) and primary (-) photon spectrum. Low-energy cutoffs and their standard errors corresponding to the total and primary photon spectrum are $E_{\text {low }}^{\text {total }}=44 \pm 6 \mathrm{keV}$ and $E_{\text {low }}^{\text {pr }}=20 \pm{ }_{20}^{10} \mathrm{keV}$, respectively. See section 3.4.2 for details on parameter error estimation.

Ramaty (1978), and Alexander and Brown (2002). Green's function for a given heliocentric angle (40 degrees for this flare) can be applied to the primary photon spectrum but as a computational convenience we modify the detector response matrix to account for the photospheric albedo. In this way it can be used straightforwardly in the forward fitting method (Kontar et al. ${ }^{3}$ ) because the result of the photon model

${ }^{3}$ http://www.astro.gla.ac.uk/users/eduard/rhessi/albedo/ 
multiplied by this new modified detector matrix is exactly the same as the result from the original detector matrix multiplied by the product of the albedo and the photon spectrum.

In addition to photospheric albedo, the RHESSI X-ray spectra generally contain a component from photons scattered in the Earth's atmosphere. The geometry of RHESSI detectors is such that most of the photons scattered in Earth atmosphere must first pass through the rear segments (see Figure 1 in Smith et al., 2002)., where the photons are effectively absorbed (the stopping depth of Ge detectors for a photon of $30 \mathrm{keV}$ is $\sim 1 \mathrm{~mm}$ ). Thus, only the photons coming from a forward semisphere can get directly into the front segments. Since RHESSI is pointed towards the Sun, the front segments are shielded by the rear ones from the Earth scattered photons all the time when RHESSI can see the Sun apart from being at the terminator. The studied flare was observed when RHESSI was far from the terminator. Therefore, a correction for the Earth albedo photons for actual zenith angle was neglected in the analysis.

The primary photon spectrum (derived under the assumption of its isotropy) together with the total photon spectrum at 08:25:22 UT (burst maximum) is shown in the top panel of Figure 2. The photon spectra differ mainly below $100 \mathrm{keV}$, the primary spectrum is slightly steeper, $\gamma_{1}^{\mathrm{pr}}=1.9$, and exhibits less flattening below $50 \mathrm{keV}$ than the total photon spectrum. Such an albedo correction significantly affects $\bar{n} V \bar{F}(E)$ mainly at electron energies below $100 \mathrm{keV}$. The bottom panel of Figure 2 shows that a forward fitted $\bar{n} V \bar{F}(E)$ consistent with the total photon spectrum has a high value of $E_{\text {low }}^{\text {total }}$, which would represent a gap in energy between the thermal and electrons producing hard Xrays. However, removing the contribution of the photospheric albedo photons results in a forward fitted $\bar{n} V \bar{F}(E)$ with $E_{\text {low }}^{\mathrm{pr}}$ close to the photon energy where the thermal part of the photon spectrum joins the non-thermal part. Consequently, no reliable dip in $\bar{n} V \bar{F}(E)$ is found.

\subsubsection{Comparison of forward fitting and inversion methods}

The mean electron flux distribution $\bar{n} V \bar{F}(E)$ corresponding to the primary photon spectrum at 08:25:22 UT derived from both the forward fitting method and the regularised inversion procedure are shown in the top panel of Figure 3. The ranges of parameter values were determined as a change $\left|\chi^{2}-\chi_{\min }^{2}\right| \sim 8.2$ while keeping the individual parameter fixed, where the level 8.2 encloses the region of joint confidence of 7 parameters with $68 \%$ confidence (Lampton, Margon and Bowyer, 1976; Press et al., 1992). Uncertainties of the forward fitted $\bar{n} V \bar{F}(E)$ were determined by multiple generations of the forward fit parameters lying within the same level of joint confidence. $\pm 1-\sigma$ limits 
of the regularised solution were found from multiple inversions of the primary photon flux data perturbed within corresponding statistical and systematic uncertainties. The inversion method was studied in detail in Kontar et al. (2004), where various modelled input spectra were analysed. Middle and bottom panels of Figure 3 represent normalised and cumulative residuals of the primary $I^{\mathrm{pr}}$ and modelled photon spectrum $I=I_{\text {th }}+I_{\text {thin }}$. Normalised residuals are defined as $r(\epsilon)=\left[I^{\mathrm{pr}}(\epsilon)-I(\epsilon)\right] / \sigma(\epsilon)$, where $\sigma(\epsilon)$ is the uncertainty in the primary photon spectrum and includes statistical plus systematic uncertainties. The error of the albedo correction method is $\sim 6 \%$, which is larger than $\sigma(\epsilon)$ but not included in it. Cumulative residuals are defined as $S(\epsilon)=1 / N \sum_{i}^{N} r_{i}$, where $N$ is the $N^{\text {th }}$ energy bin used in fitting, and can be used to assess clustering of residuals in certain energy ranges (Piana et al., 2003). Normalised residuals of the best fit are limited to a 3- $\sigma(\epsilon)$ interval. Cumulative residuals show minor clustering in the photon energy range $20-80 \mathrm{keV}$ but they are well below $\pm 1-\sigma$ limits of random walk $(\sigma=1 / \sqrt{N})$, see full lines in bottom panel of Figure 3 . Both normalised and cumulative residuals also indicate that assumed systematic uncertainties (5\% in each energy bin) are overestimated. Adopting a lower value of $3 \%$ results in residuals distributed as expected for Gaussian statistics with $\sim 1 / 3$ of them above the $1-\sigma$ level, but it does not modify $\bar{n} V \bar{F}(E)$ significantly.

The best forward fit parameters for the primary spectrum are: emission measure $E M=\left(1 \pm{ }_{0.4}^{2.0}\right) \times 10^{49} \mathrm{~cm}^{-3}$, temperature $T=(1.3 \pm$ $0.2) \mathrm{keV}, E_{\text {low }}=\left(20 \pm{ }_{20}^{10}\right) \mathrm{keV}, \delta_{1}=0.4 \pm 0.2, \delta_{2}=3.2 \pm{ }_{0.2}^{0.3}, E_{\text {break }}=$ $\left(240 \pm{ }_{30}^{40}\right) \mathrm{keV}, \bar{n} V \bar{F}=\left(17 \pm{ }_{0.8}^{3.0}\right) \times 10^{55}$ electrons $\mathrm{cm}^{-2} \mathrm{~s}^{-1}$, where $\bar{F}$ is $\bar{F}(E)$ integrated from $E_{\text {low }}$ to $E_{\text {high }}$. Several parameters $\left(E M, E_{\text {low }}\right.$, $\bar{n} V \bar{F})$ have significantly different, by a factor of 5 , estimates of high and low $\sigma$. This behaviour is mainly due to the low value of $\delta_{1}$ because a decrease in $E_{\text {low }}$ and consequent increase in $\bar{n} V \bar{F}$ does not result in significant change in the modelled primary photon spectrum. The primary photon spectrum is therefore, within uncertainties, consistent also with a mean electron flux distribution function without a low-energy cutoff as derived from the forward fitting method. Large uncertainties in $E M$ are caused by the small number of data points in the thermal component of the spectrum (see top panel of Figure 2).

The mean electron flux distribution deduced from the forward fitting agrees within uncertainties with the regularised solution up to $\sim 200 \mathrm{keV}$. The discrepancy at higher energies is caused by different high-energy cutoffs in the forward fitting and inversion methods. The regularised solution was cut at $0.5 \mathrm{MeV}$ whereas the double power-law $\bar{F}(E)$ extended to $E_{\text {high }}=7 \mathrm{MeV}$. The large uncertainties between 20 and $40 \mathrm{keV}$ shown in Figure 3 result from the small number of electrons 


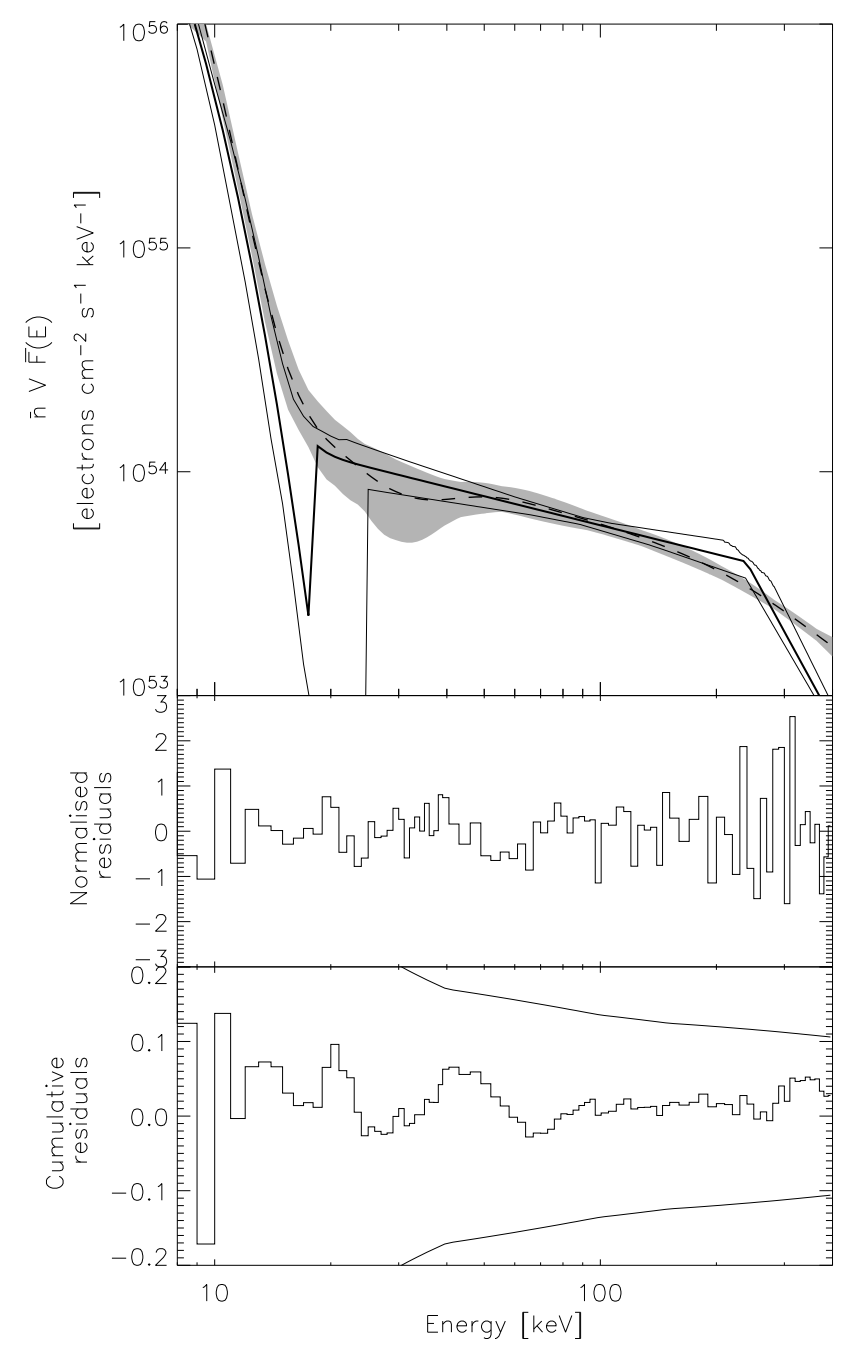

Figure 3. Top panel shows comparison of $\bar{n} V \bar{F}(E)$ deduced from forward fitting (-) and inversion (- - -) method for primary photon spectrum at 08:25:22 UT. Shaded area represents $\pm 1-\sigma$ limits on the regularised solution, thin full lines correspond to $68 \%$ confidence strip for the forward fit solution. Middle and bottom panels show normalised and cumulative residuals of the primary and modelled photon spectrum for the forward fitting method. Thin lines in the bottom panel represent $\pm 1-\sigma$ limits for a random walk process.

in this energy range and the fact that the photons are primarily from higher energy electrons. A similar argument can be used to explain the deviation between the forward fit and regularised solutions above 100 $\mathrm{keV}$. The high value of $E_{\text {high }}$ used in the forward-fit solution introduces 


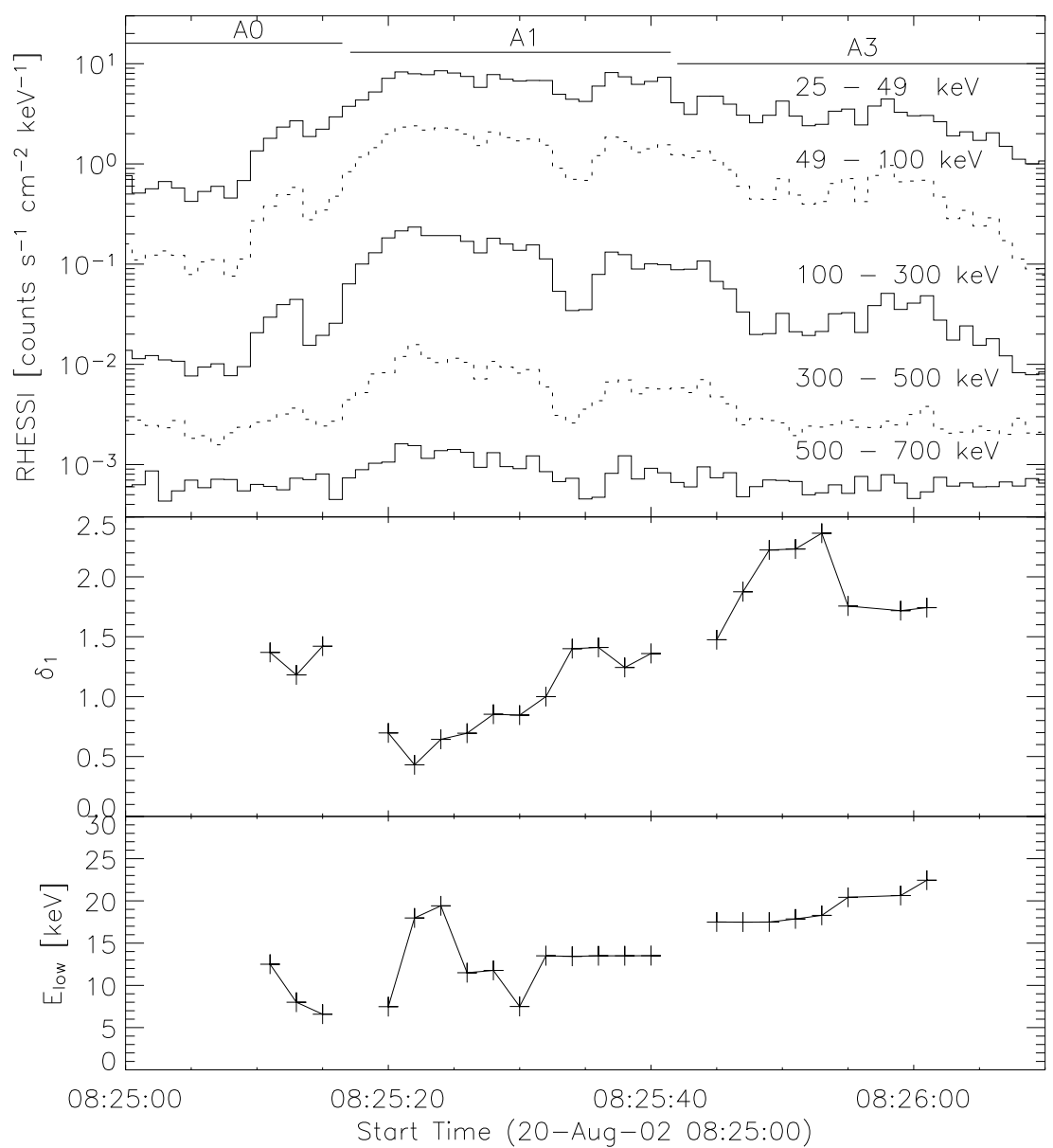

Figure 4. Time evolution of RHESSI X-ray count flux (1-s time resolution) in five energy channels, the power-law index $\delta_{1}$, and the low-energy cutoff $E_{\text {low }}$ of the mean electron flux distribution $\bar{n} V \bar{F}(E)$. Attenuator states A0, A1, and A3 are indicated by lines at the top of RHESSI data plot. For display purposes the RHESSI count flux at the $300-500 \mathrm{keV}$ band was scaled by a factor of 2 .

a contribution to the photon flux above $100 \mathrm{keV}$ from the higher energy electrons not included in the regularised electron spectrum.

In the case of the total photon spectrum, i.e. without the albedo correction, the regularised solution indicates a dip within 1- $\sigma$ limits in the energies below $\sim 30 \mathrm{keV}$ where the forward fitted solution resulted in a gap above the thermal component in $\bar{n} V \bar{F}(E)$, see Figure 2 .

\subsubsection{Time evolution}

Figure 4 shows the time evolution of derived parameters $\delta_{1}$ and $E_{\text {low }}$ of the mean electron flux distribution corresponding to the primary 
photon spectra. The power-law index $\delta_{1}$ decreases to its minimum of $0.4 \pm 0.2$ at the burst maximum, 08:25:22 UT, and its time history generally shows a soft-hard-soft pattern between the photon flux and hardness of the spectra (e.g. Fletcher and Hudson, 2002). Low values of $\delta_{1}$ indicate a flat electron flux spectrum and this is confirmed by the regularised inversion method. The low-energy cutoff $E_{\text {low }}$ lies within or close to the energies where the thermal component dominates $\bar{n} V \bar{F}(E)$ distribution. Standard errors are of the order of the $E_{\text {low }}$ values. Therefore, no reliable dips or gaps in $\bar{n} V \bar{F}(E)$ throughout the time interval could be established. Our analysis shows that the low-energy cutoffs above the energies of the thermal component are likely to be the result of the contribution of photospheric albedo photons and do not exist in real mean electron spectra.

\section{H $\alpha$ data analysis and magnetic field topology}

To study the influence of the accelerated electrons on the $\mathrm{H} \alpha$ emission, we used full-disk $\mathrm{H} \alpha$ images covering the whole interval of the flare. The images were acquired using a filter with $0.07 \mathrm{~nm}$ FWHM at Kanzelhöhe Solar Observatory in Austria. The time resolution of the data is $3 \mathrm{~s}$ and the spatial resolution is $2.2 \mathrm{arcsec} / \mathrm{pixel}$. The position of the solar centre and the spatial resolution were determined using the routines provided by A. Veronig. Details on the method can be found in Veronig et al. (2000).

The intensities from the flare site were scaled by the averaged quietSun area intensity $\mathrm{I}_{\mathrm{q}}$ for every image separately. The brightest parts of the flare area beginning at 08:25:20 UT are distorted by the saturation of detected intensities. The saturated pixels are displayed with horizontal lines in the $\mathrm{H} \alpha$ images (Figures 5, 6, and 9).

The data set was further spatially coaligned using a technique developed for TRACE and EIT data (Gallagher ${ }^{4}$ ). The cross-correlation of the $\mathrm{H} \alpha$ data set was done in the area of the nearby sunspot with respect to the chosen $\mathrm{H} \alpha$ image. That sunspot area was not affected by the flare. Therefore, we assumed no time change of the $\mathrm{H} \alpha$ intensities during the analysed time interval in that area. The positional uncertainty of the $\mathrm{H} \alpha$ images was estimated to be of the order of 1 pixel $(2.2$ arcsec $)$.

\subsection{Spatial Evolution}

The evolution of the hard X-ray sources overlayed with the $\mathrm{H} \alpha$ emission and MDI magnetic neutral lines is displayed in Figures 5 and 6 . The

\footnotetext{
${ }^{4}$ http://hesperia.gsfc.nasa.gov/ ptg/trace-align/index.html
} 

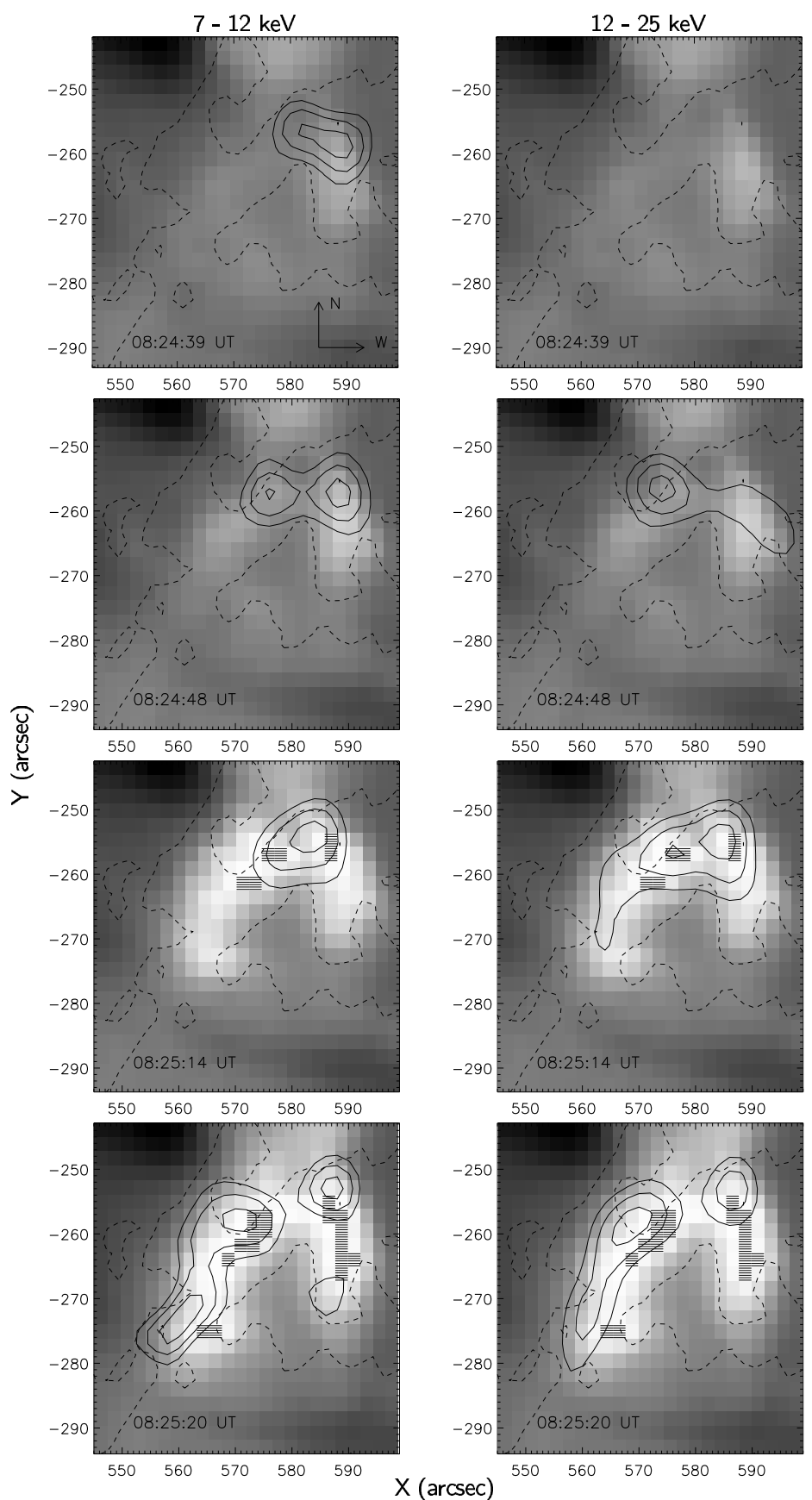

Figure 5. Time and spatial evolution of $\mathrm{H} \alpha$ intensities (grey scale) and X-ray emission (full line contours) in the $7-12 \mathrm{keV}$ (first column) and $12-25 \mathrm{keV}$ (second column) energy ranges. Contours correspond to 50,70 , and $90 \%$ of maximum of each RHESSI CLEAN image. There were no detectable X-ray sources in the $12-25 \mathrm{keV}$ energy range at 08:24:39 UT (see the image in the top right corner). MDI magnetic neutral lines are plotted as dashed lines. The saturated $\mathrm{H} \alpha$ pixels are displayed with horizontal lines. 

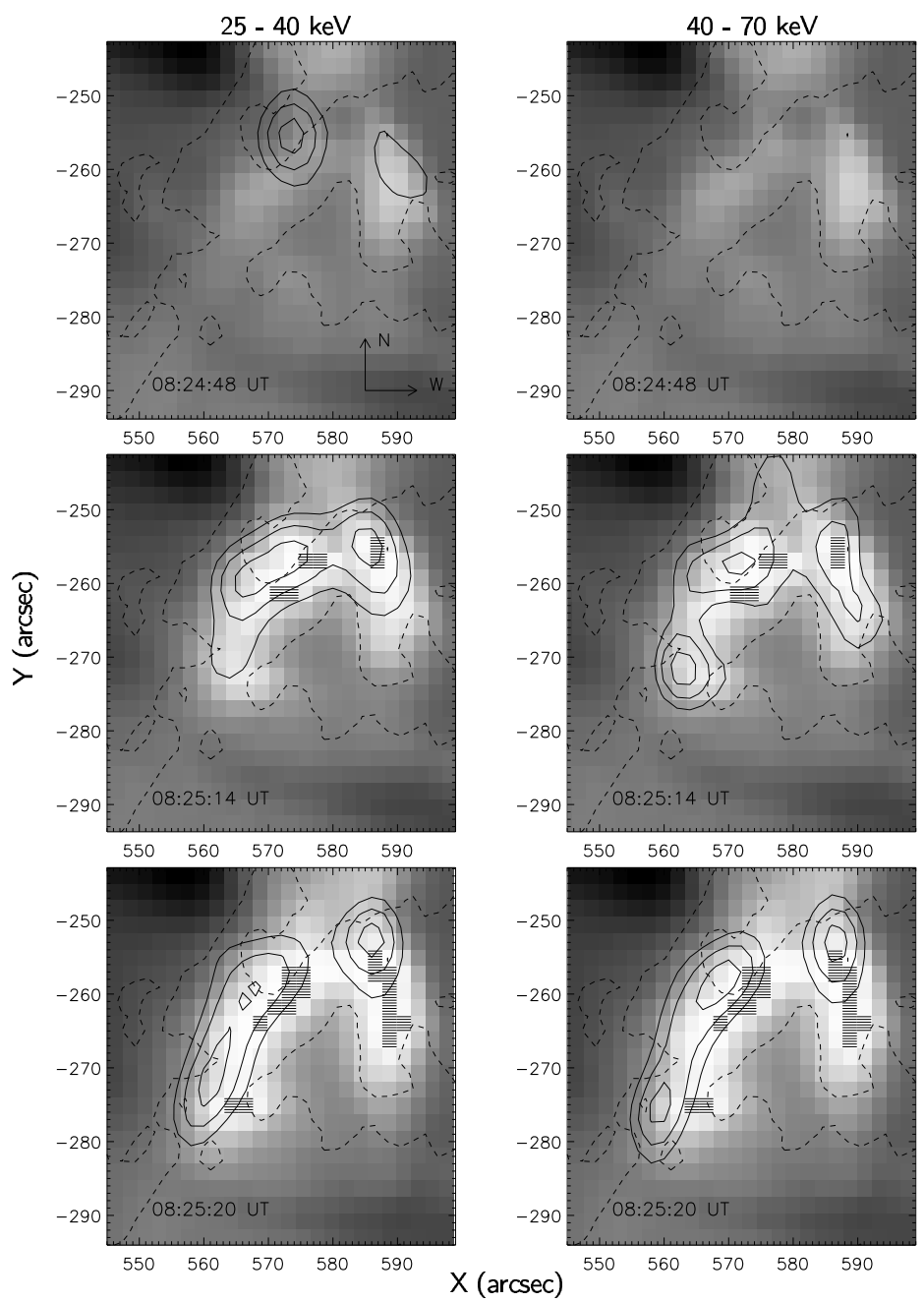

Figure 6. Time and spatial evolution of $\mathrm{H} \alpha$ intensities (grey scale) and X-ray emission (full line contours) in the $25-40 \mathrm{keV}$ (first column) and $40-70 \mathrm{keV}$ (second column) energy ranges. Contours correspond to 50,70 , and $90 \%$ of maximum of each RHESSI CLEAN image. There were no detectable X-ray sources in the $40-70 \mathrm{keV}$ energy range at 08:24:48 UT (see the image in the top right corner). MDI magnetic neutral lines are plotted as dashed lines. The saturated $\mathrm{H} \alpha$ pixels are displayed with horizontal lines.

RHESSI flux was accumulated for 1 spin period (4.141 s). The $\mathrm{H} \alpha$ images correspond to the middle of the RHESSI imaging time range. Due to the saturation of the $\mathrm{H} \alpha$ intensities, the spatial evolution in the flare area is shown to 08:25:20 UT only.

At the very beginning of the flare (at 08:24:39 UT, Figure 5), a single X-ray source is detected. Then it splits into two (at 08:24:48 UT, Fig- 
ures 5 and 6) and appears to be moving eastwards. Later at 08:25:14 UT, the X-ray emission starts to shift southwards and the sources split even into three components detectable in the energy range $40-70 \mathrm{keV}$. There is a difference in the positions of hard X-ray sources at lower and higher energy ranges detected at the same time. The X-ray sources at higher energies tend to be located more to the south than the lower energy $\mathrm{X}$-ray sources (compare the positions of the sources in different energy bands at 08:25:14 UT and 08:25:20 UT in Figures 5 and 6). Most of Xray source maxima at energies above $12 \mathrm{keV}$ are not spatially coincident with the brightest parts of the $\mathrm{H} \alpha$ emission. Rather they tend to be located near the north and eastern edges of the bright $\mathrm{H} \alpha$ kernels. The $\mathrm{H} \alpha$ kernels form a semi-circle whose inner boundary is located near the magnetic neutral line.

\subsection{Magnetic topology}

To understand better the magnetic field topology near the flare site, the extrapolation of magnetic field in the current-free approximation (Démoulin et al., 1997) was made using the MDI magnetogram observed at 08:12:30 UT (see Figure 7).

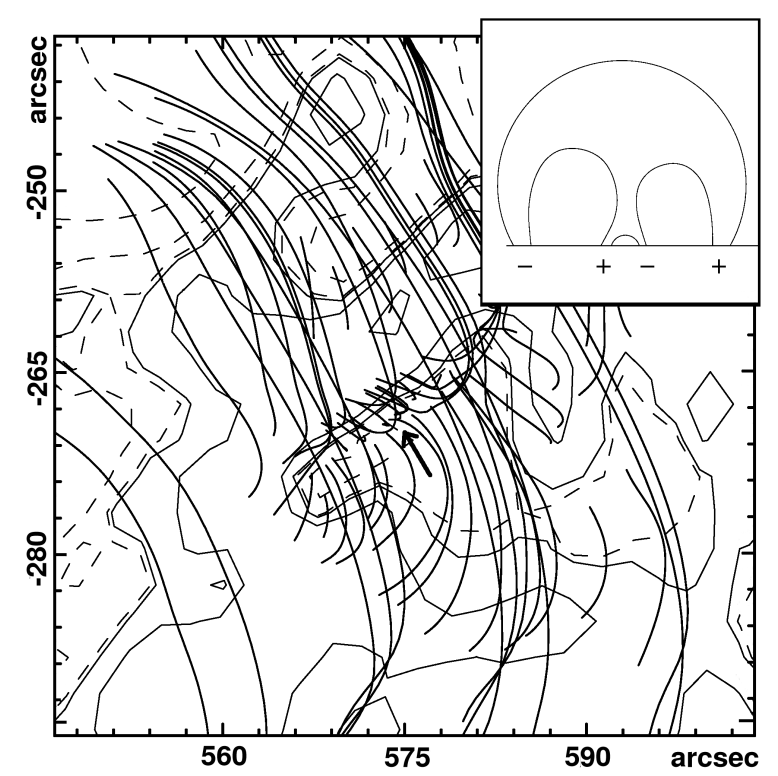

Figure 7. Magnetic field lines, extrapolated in the current-free approximation at 08:12:30 UT (full thick lines), revealing the quadrupolar topology near the flare site. The isolines of the north and south magnetic polarities $( \pm 10, \pm 100$, and $\pm 400 \mathrm{G}$ ) are expressed by thin full and dashed lines, respectively. A cut through the region of the neutral magnetic line indicated by the arrow is shown in the sketch in the top right corner (here sizes of loops are not to scale). 
The most interesting structure, indicated by an arrow in Figure 7, is located along the magnetic neutral line, which is delineated by the above mentioned semi-circular $\mathrm{H} \alpha$ ribbon. Namely, this structure has a quadrupolar character in the sense of papers by Uchida (1996) and Hirose and Uchida (2002), i.e. low altitude loops in the close vicinity of the neutral line are between magnetic field lines with the opposite orientation and all is covered by extended overlying loops.

The preflare activity in the $1550 \AA$ TRACE band was observed directly in the centre of the quadrupolar structure (i.e. above the magnetic neutral line) at 08:12:20 UT. However, no 3 - $6 \mathrm{keV}$ X-ray emission was detected by RHESSI from this flare site at this time.

Comparing Figures 5,6 and 7, it can be seen that the flare started at the north end of this quadrupolar structure. Then the flare kernels appeared at the north-east boundary of the structure and the flare ends at the southern extremity of the quadrupolar structure. We also note that the $\mathrm{H} \alpha$ ribbon and X-ray sources are shifted $\sim 4$ arcsec from the quadrupolar configuration of the extrapolated magnetic field lines.

\subsection{Time Derivative of $\mathrm{H} \alpha$ intensities}

We assumed that the locations of the optical flare that are directly affected by the electron beams might be recognised as areas of abrupt changes of the $\mathrm{H} \alpha$ intensity, $I_{\mathrm{H} \alpha}$, on a time scale of the order of seconds. This assumption is supported e.g. by Trottet et al. (2000), who found correlation between hard X-ray flux and $\mathrm{H} \alpha$ intensities on time scales from seconds to tens of seconds. Therefore, the time derivative of $I_{\mathrm{H} \alpha}$ may provide a technique to find such areas.

In order to suppress the effects of seeing and/or incorrect positions, $\mathrm{H} \alpha$ intensities were averaged over a $3 \times 3$ pixel area and the resulting lightcurves were smoothed by a 3-point box car (9 s time resolution). Such smoothing was necessary to distinguish between the real changes and those which occurred randomly throughout the whole flare area. The effects of the averaging and smoothing are shown in Figure 8. Due to the saturation of $\mathrm{H} \alpha$ data beginning at 08:25:20 UT, the time derivative of $\mathrm{H} \alpha$ intensity, $\mathrm{d} I_{\mathrm{H} \alpha} / \mathrm{d} t$, was studied in the time interval 08:24:33 - 08:25:20 UT (see also the label in Figure 1). The maximum of $\mathrm{d} I_{\mathrm{H} \alpha} / \mathrm{d} t$ was $0.097 \mathrm{I}_{\mathrm{q}} \mathrm{s}^{-1}$. A threshold, $\mathrm{d} I_{\mathrm{H} \alpha} / \mathrm{d} t=0.03 \mathrm{I}_{\mathrm{q}} \mathrm{s}^{-1}$, was estimated by fitting $\mathrm{d} I_{\mathrm{H} \alpha} / \mathrm{d} t$ distribution outside of the flare area with the Gauss distribution. The threshold corresponds to 10 standard deviations from the mean of the fit and was adopted as the level of the reliable value of $\mathrm{d} I_{\mathrm{H} \alpha} / \mathrm{d} t$ not affected by noise or seeing.

The locations of $\mathrm{d} I_{\mathrm{H} \alpha} / \mathrm{d} t>0.03 \mathrm{I}_{\mathrm{q}} \mathrm{s}^{-1}$ are displayed on Figure 9 together with the RHESSI CLEAN images in the $25-40 \mathrm{keV}$ and 40 - 


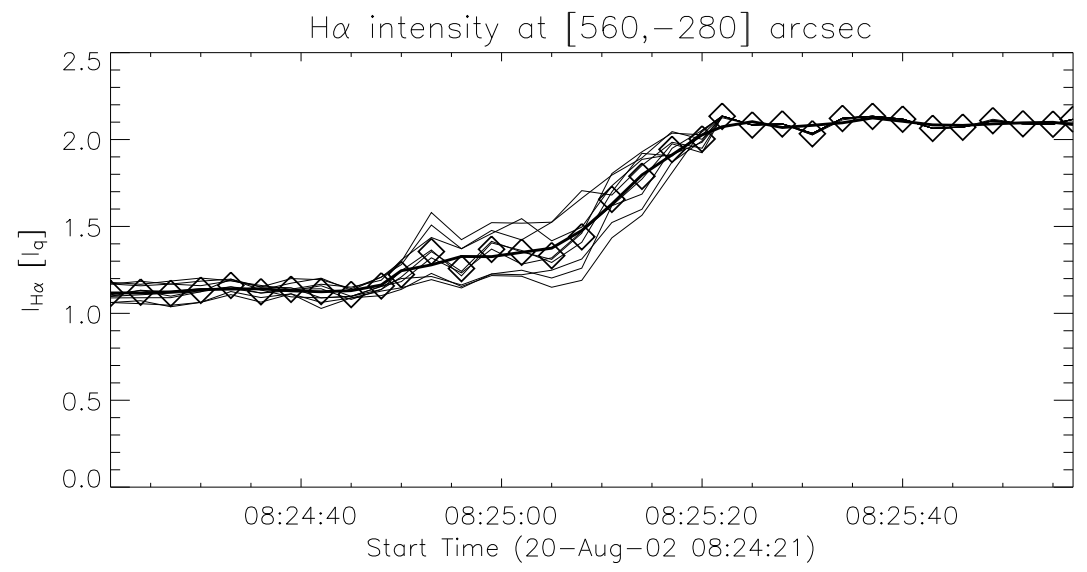

Figure 8. Averaging and smoothing effects of $I_{\mathrm{H} \alpha}$ at the location $[560,-280]$ arcsec revealing the largest derivative of $I_{\mathrm{H} \alpha}$ (see Figure 9). Thin lines show observed time histories at $3 \times 3$ pixel area, $\diamond$ correspond to the average over that area, and the thick line is the resulting time history smoothed by 3-point box car. Intensities are scaled by the quiet Sun intensity $I_{\mathrm{q}}$.

$70 \mathrm{keV}$ energy bands. X-ray sources in the 40 - $70 \mathrm{keV}$ energy band are not shown in Figure 9(a) since no source at this energy band was detectable at that time.

These energy bands were chosen for comparison with $\mathrm{d} I_{\mathrm{H} \alpha} / \mathrm{d} t$ for two reasons. First, they were above the energies where the thermal component dominates X-ray emission, i.e. $\gtrsim 12 \mathrm{keV}$. Secondly, the electrons bombarding the chromosphere need to have an energy greater than $25 \mathrm{keV}$ to penetrate into the layers of $\mathrm{H} \alpha$ formation, e.g. in the F1 flare model by Machado et al. (1980). The RHESSI CLEAN images were reconstructed from data accumulated in 1 spin period. The centre of each RHESSI time interval corresponds to the observational times of $\mathrm{H} \alpha$ and $\mathrm{d} I_{\mathrm{H} \alpha} / \mathrm{d} t$.

The sites of the fast $\mathrm{H} \alpha$ intensity changes located in the northern part of the flare lie on the boundaries or inside of X-ray sources in the $25-40 \mathrm{keV}$ and/or $40-70 \mathrm{keV}$ energy range (see Figures 9(b) and (c)). However, we have found other areas that reveal abrupt changes in $\mathrm{H} \alpha$ intensity but are located well away from X-ray emission (e.g. Figure 9(a)). Some of these areas even precede the position of X-ray emission as it moves to the south in time (Figures 9(c) and (d)). The difference in the locations of $\mathrm{d} I_{\mathrm{H} \alpha} / \mathrm{d} t$ and the X-ray sources cannot be explained by a spatial correlation with the X-ray sources at lower or higher energies. There is no significant shift of the X-ray sources in the energy ranges from $3-7 \mathrm{keV}$ to $7-12 \mathrm{keV}$ or from $40-70 \mathrm{keV}$ to 

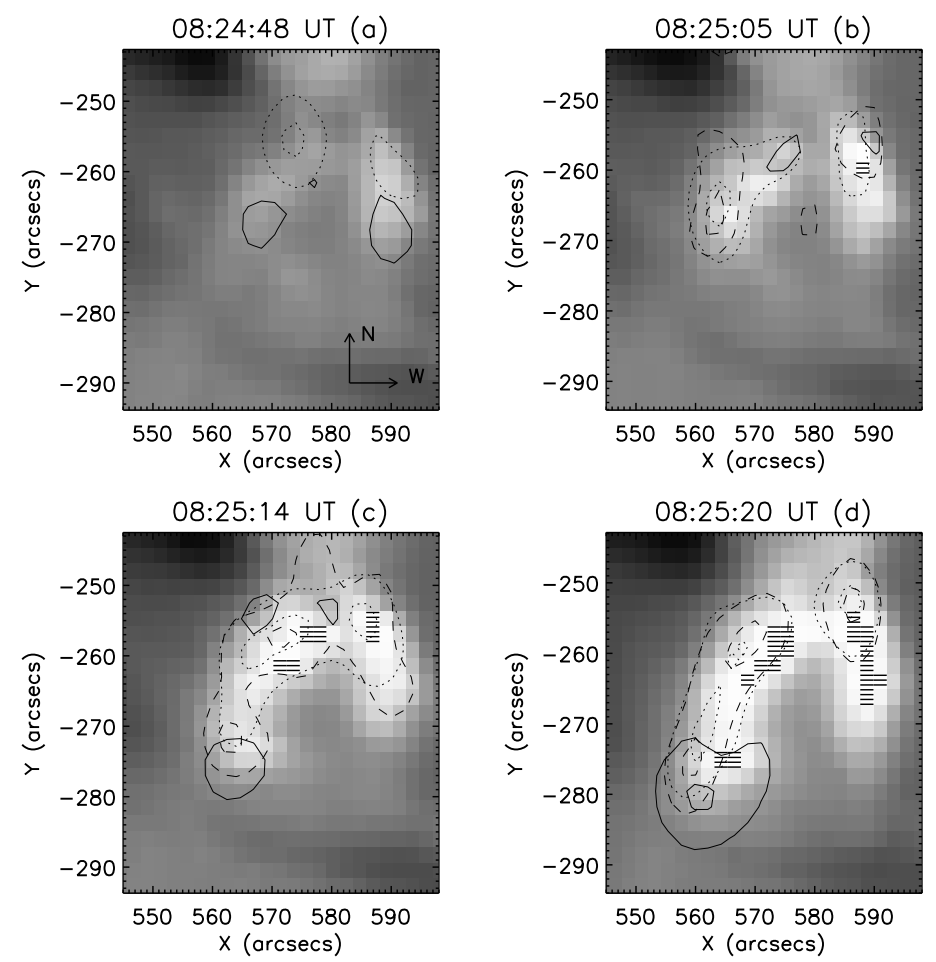

Figure 9. Locations of time derivative of $\mathrm{H} \alpha$ intensities (full line contours) overlayed on $\mathrm{H} \alpha$ images (grey scale). Contours correspond to $0.04 \mathrm{I}_{\mathrm{q}} \mathrm{s}^{-1}$ and $0.09 \mathrm{I}_{\mathrm{q}} \mathrm{s}^{-1}$ of $\mathrm{d} I_{\mathrm{H} \alpha} / \mathrm{d} t$. RHESSI CLEAN images at $25-40 \mathrm{keV}$ and $40-70 \mathrm{keV}$ are plotted as dotted and dashed contours at 50 and $90 \%$ of maximum of each image. The saturated $\mathrm{H} \alpha$ pixels are displayed with horizontal lines.

higher energy bands; the sources at energies from 7 to $25 \mathrm{keV}$ are not located closer to the $\mathrm{d} I_{\mathrm{H} \alpha} / \mathrm{d} t$ (see Figures 5 and 6 ) either.

The fact that at 08:25:20 UT there are no locations of $\mathrm{d} I_{\mathrm{H} \alpha} / \mathrm{d} t>$ $0.03 \mathrm{I}_{\mathrm{q}} \mathrm{s}^{-1}$ along the whole flare area may be the result of the saturation of the $\mathrm{H} \alpha$ data at this time (areas covered by horizontal lines in Figure 9). Although the described analysis technique does not eliminate the saturated pixels, they only affect the adjacent pixels and cause a decrease in $\mathrm{d} I_{\mathrm{H} \alpha} / \mathrm{d} t$.

\section{Discussion}

The photon spectrum at the burst maximum of the August 20, 2002 flare is, to our knowledge, the flattest $\left(\gamma_{1}=1.8\right)$ yet detected by RHESSI with its high energy resolution. To eliminate any distortion of the spectra, the background subtraction also included the estimation 
of the electron precipitation component, which has been done for the RHESSI data for the first time. The solar origin of such an unusually flat spectrum was confirmed by a comparison with the spectrum constructed from the RHESSI PIXON images.

Using a new method of the photospheric albedo correction independent of the spectral form of the primary spectrum, we have proved the importance of the photospheric albedo correction in the case of the analysed flare. We have shown that in this case of flat X-ray spectra, the photospheric albedo significantly affects the determination of the mean electron flux distribution $\bar{n} V \bar{F}(E)$, mainly the value of the low-energy cutoff. The contamination of the flare spectra by the photospheric albedo photons may result in $\operatorname{dips}$ in $\bar{n} V \bar{F}(E)$ indicated both by the forward fitting and inversion methods. Such dips could be misinterpreted as spectral features inherent to the acceleration/propagation processes of the energetic particles in the solar flares.

Flares of flat hard X-ray spectra $(\gamma \leq 2)$ were previously analysed e.g. by Nitta, Dennis, and Kiplinger (1990) and Fárník, Hudson, and Watanabe (1997). All of these flares have weak thermal components (GOES class M3 or lower) but intense non-thermal components up to several hundred $\mathrm{keV}$ with a flat part extending down to $\sim 10 \mathrm{keV}$. As was shown here, such photon spectra may correspond to a flat electron flux distribution with the low-energy cutoff close to the energy of the thermal electrons.

For the flares with spectral index around 3 it is the low-energy cutoff which determines the total energy content in the flare. This happens when the largest contribution comes from the low energy particles. However, for the flares with flat spectra $\delta_{1} \lesssim 1$ it is not the case. Assuming the collisional thick-target scenario (Brown 1971), one can estimate the energy flux of the non-thermal electrons injected into the plasma. In this model, the injected electron flux distribution $\mathcal{F}\left(E_{0}\right)$ [electrons s${ }^{-1} \mathrm{kev}^{-1}$ ] is related to $\bar{F}(E)$ of Equation (2) as (Brown and Emslie, 1988)

$$
\mathcal{F}\left(E_{0}\right) \sim \bar{F}\left(E_{0}\right) E_{0}^{-2}(\mathrm{~d} \ln \bar{F}(E) / \mathrm{d} \ln E+1)_{E=E_{0}} \sim \bar{F}\left(E_{0}\right) E_{0}^{-2} .
$$

Relative change of the non-thermal energy flux $\mathfrak{F}_{E_{\text {low }}}\left[\mathrm{keV} \mathrm{s}^{-1}\right]$ can be expressed

$$
\mathfrak{F}_{E_{\text {low }}^{\prime}} / \mathfrak{F}_{E_{\text {low }}} \sim\left(E_{\text {low }}^{\prime} / E_{\text {low }}\right)^{-\delta_{1}} \quad \mathfrak{F}_{E_{\text {low }}}=\int_{E_{\text {low }}}^{E_{\text {high }}} \mathcal{F}\left(E_{0}\right) E_{0} \mathrm{~d} E_{0} .
$$

Adopting the spectral index $\delta_{1}=0.4$ and the low-energy cutoffs, $E_{\text {low }}^{\prime}=$ $10 \mathrm{keV}, E_{\text {low }}=30 \mathrm{keV}$, consistent with the inferred flat spectrum, the ratio is $\sim 2$. Thus, due to the flatness of $\bar{n} V \bar{F}(E)$, the low values of the 
low-energy cutoff do not cause a significant increase in the non-thermal energy flux.

The flat distribution function of non-thermal electrons, especially at low energies, implies that its derivative in the energy as well as in the velocity space is close to zero or even positive. Such a distribution function can be formed directly in the acceleration region (e.g. by the acceleration in the direct electric field - Holman, 1985) and/or modified by propagation effect, particle collisions in dense plasma, and by interactions of accelerated electrons with plasma waves (Melnik and Kontar, 2003). If the plasma waves are generated, then the radio emission can be observed at high frequencies, as in our case. However, detailed conditions in the radio source needed to confirm this idea are not known to us.

Our analysis of the characteristics of the hard X-ray sources and the $\mathrm{H} \alpha$ and radio emission revealed several facts about the flare process. First, the presented flare was weak in the metric range during the whole event, except for the short-lasting relativistic type III burst (radio manifestation of relativistic electrons; Klassen, Karlický, and Mann, 2003). It resembles the March 6, $1989 \gamma$-ray flare, which started as silent in radio waves below $800 \mathrm{MHz}$. Similarly to Rieger, Treumann, and Karlický (1999), we explain the lack of metric radio emission by closed geometry of the magnetic structure and a low altitude of the flare process. Additionally, the high value of the turn-over frequency, 19.6 - $50 \mathrm{GHz}$, of the radio emission indicates a strong magnetic field or a large depth of the emitting source (Benka and Holman, 1992), thus supporting this conclusion. Furthermore, the coalignment of the $\mathrm{H} \alpha$ and hard X-ray images with the magnetic field extrapolation shows that both the $\mathrm{H} \alpha$ kernels and the hard X-ray sources are located at the footpoints of the low altitude magnetic loops which are the part of the quadrupolar structure (Figure 7).

Second, our results concerning the time and spatial correlation of the hard X-ray sources and $\mathrm{H} \alpha$ emission are partly in agreement with the prediction of solar flare models. We have found that most of the $\mathrm{X}$-ray emission maxima, mainly those at the energies above $12 \mathrm{keV}$ (corresponding to the X-ray emission of the non-thermal electrons), tend to be located on the northern and eastern edges of the $\mathrm{H} \alpha$ emission (see Figures 5 and 6 ). This is consistent with the expectation that Xray sources should be located on the external edges of $\mathrm{H} \alpha$ ribbons and similar to those reported by Czaykowska et al. (1999).

Flare models also suggest that fast changes of $\mathrm{H} \alpha$ intensities might be located at positions of X-ray sources, i.e. at the areas which are bombarded by the suprathermal electrons. In some cases, this suggestion is confirmed by our analysis. However, at some times the positions 
of the fast changes of $\mathrm{H} \alpha$ intensities do not spatially coincide with the X-ray sources (see southern locations of $\mathrm{d} I_{\mathrm{H} \alpha} / \mathrm{d} t$ in the bottom row of Figure 9). We propose that such $\mathrm{H} \alpha$ brightenings might be caused either by thermal conduction from a hot plasma located above, or by soft X-ray irradiation. Using the lowest energy ranges of RHESSI (3 $7 \mathrm{keV}$ ), we tried to find such a hot plasma at these places, but without any success. However, we must point out that the time resolution of $\mathrm{d} I_{\mathrm{H} \alpha} / \mathrm{d} t$ was $9 \mathrm{~s}$. Therefore to confirm the existence of these areas, more analyses and better quality data are needed.

Finally, the time evolution of the positions of the hard X-ray sources may indicate the movement of the reconnection site. This can be seen e.g. at 08:25:14 UT (Figures 5 and 6) when the hard X-ray sources of different energies are distributed along the $\mathrm{H} \alpha$ ribbon in such a way that the sources of higher energy are located further to the southeast direction. Such difference in the positions could be expected in a single flare loop when it would correspond to the height distribution of the hard X-ray sources along the loop as found in Aschwanden, Brown, and Kontar (2002). However, this is not the case here because the extrapolation of the magnetic field shows no magnetic loop along the $\mathrm{H} \alpha$ ribbon. The displacement of the $\mathrm{H} \alpha$ ribbon and X-ray sources from the quadrupolar configuration is not due to the positional errors in the $\mathrm{H} \alpha$, MDI, and RHESSI images, which are a factor of 2 and 4 smaller, respectively. The offset could be caused by neglecting the electric currents in the current-free extrapolation. Although the linear force-free extrapolations show similar results, we cannot exclude the presence of localised currents which deform the magnetic field structure.

\section{Conclusions}

We have demonstrated that the influence of the photospheric albedo on solar flare X-ray spectra must be considered in order to correctly assess the models of electron acceleration and propagation and generation of hard X-ray emission. The inferred flat mean electron flux distribution could be the result of the acceleration process and/or mod-

ified by particle-wave interactions. This explanation, however, remains speculative. The accumulated information from the multi-wavelength observations of this flare shows that the flare process took place in the low altitude magnetic loops. Our analysis of $\mathrm{H} \alpha$ emission may indicate that the response of the lower atmosphere to the flare energy release is not restricted to the sites of propagation of the accelerated electrons. 


\section{Acknowledgements}

One of the authors, JK, would like to give special thanks to the RHESSI team at GSFC for their help and support during her stay there. We are thankful to W. Otruba (Kanzelhöhe Solar Observatory, Austria) and A. Veronig for providing $\mathrm{H} \alpha$ data and for valuable discussion on their analysis. We also thank T. Metcalf for help with the PIXON reconstruction, D. Zarro for preprocessing TRACE data, and P. Démoulin for providing his code for the magnetic field extrapolation. Furthermore, we thank P. Messmer and T. Lüthi for the Zürich and Bern radio data, respectively.

This work was supported by NATO Science Fellowships Programme, the contract NAS5 - 01160, the grants IAA3003202 and IAA3003203 of the Academy of Sciences of the Czech Republic, the grants 205/02/0980 and 205/04/0358 of the Grant Agency of the Czech Republic, and the projects K2043105 and AV0Z10030501 of the Astronomical Institute.

\section{References}

Aschwanden, M. J., Brown, J. C., and Kontar, E. P.: 2002, Solar Phys., 210, 383.

Alexander, D. and Metcalf, T. R.: 1997, ApJ, 489, 422.

Alexander, R. C. and Brown, J. C.: 2002, Solar Phys., 210, 407.

Asai, A., Masuda, S., Yokoyama, T., Shimojo, M., Isobe, H., Kurokawa, H., and Shibata, K.: 2002, ApJL, 578, L91

Bai, T. and Ramaty, R.: 1978, ApJ, 219, 705.

Benka, S. G. and Holman, G. D.: 1992, ApJ, 391, 854.

Brown, J. C.: 1971, Solar Phys., 18, 489.

Brown, J. C. and Emslie, A. G.: 1988, ApJ, 331, 554.

Brown, J. C., Emslie, A. G. and Kontar, E. P.: 2003, ApJ, 595, L115

Cargill, P. J. and Priest, E. R.: 1983, ApJ, 266, 383

Canfield, R. C. and Gayley, K. G.: 1987, ApJ, 363, 1999

Czaykowska, A., de Pontieu, B., Alexander, D., and Rank, G.: 1999, ApJ, 521, L75.

Démoulin, P., Bagalá, L. G., Mandrini, C. H., Hénoux, J. C., and Rovira, M. G.: 1997, Astron. Astrophys., 325, 305.

Dennis, B. R.: 1985, Solar Phys., 100, 465.

Dennis, B. R. and Schwartz, R. A.: 1989, Solar Phys., 121, 75.

Fárník, F., Hudson, H., and Watanabe, T: 1997, Astron. Astrophys., 320, 620

Fletcher, L. and Hudson, H.: 2002, Solar Phys., 210, 307.

Haug, E.: 1997, Astron. Astrophys., 326, 417.

Heinzel, P.: 1991, Solar Phys., 135, 65.

Heyvaerts, J., Priest, E. R., and Rust, D. M.: 1977, ApJ, 216, 123.

Hirose, S. and Uchida, Y.: 2002, in P. C. H. Martens and D. P. Cauffman (eds), Multi-wavelenght observations of coronal structure and dynamics, Yohkoh 10th Anniversary Meeting, p. 181.

Holman, G. D.: 1985, ApJ, 293, 584. 
Holman, G. D., Sui, L., Schwartz, R. A., and Emslie, A. G.: 2003, ApJ, 595, L97.

Hurford, G. J., Schmahl, E. J., Schwartz, R. A., Conway, A. J. et al.: 2002, Solar Phys., 210, 61.

Johns, Ch. M. and Lin, R. P.: 1992, Solar Phys., 137, 121.

Klassen, A., Karlický, M., and Mann, G.: 2003, Astron. Astrophys., 410, 307.

Karlický, M.: 1997, Space Science Rev., 81, 143.

Kontar, E. P., Piana, M., Massone, A. M., Emslie, A. G., and Brown, J. C.: 2004, Solar Phys., 225, 293.

Kontar, E. P., Emslie, A. G., Piana, M., Massone, A. M., and Brown, J. C.: 2005a, Solar Phys., 226. 317.

Kontar, E. P., MacKinnon, A. L., Schwartz, R. A., and Brown, J. C.: 2005b, ApJ, submitted.

Lampton, M., Margon, B., and Bowyer, S.: 1976, ApJ, 208, 177.

Lin, R. P. and Schwartz, R. A.: 1987, ApJ, 312, 462.

Lin, R. P. et al.:, 2002, Solar Phys., 210, 3.

Machado, M. E., Avrett, E. H., Vernazza, J. E., and Moyes, R. W.: 1980, Astron. Astrophys., 242, 336.

Melnik, V.N. and Kontar, E.P.: 2003, Solar Phys., 215, 335.

Metcalf, T.'R., Hudson, H. S., Kosugi, T., Puetter, R. C., and Piña, R. K.: 1996, ApJ, 466, 585.

Nitta, N., Dennis, B. R., and Kiplinger, A. L.: 1990, ApJ, 353, 313

Piana, M., Massone, A. M., Kontar, E. P., Emslie, A. G., Brown, J. C., and Schwartz, R. A.: 2003, ApJ, 595, L127.

Poquerusse, M.: 1994, Astron. Astrophys. 286, 611.

Press, W. H., Teukolsky, S. A., Vetterling, W. T., and Flannery, B. P.: 1992, Numerical recepies in $C$ : the art of scientific computing, 2nd ed., Cambridge University Press, p. 684

Puetter, R. C. and Piña, R. K.: 1994, Experimental Astronomy, 3, 293.

Rieger, E., Treumann, R. A., and Karlický, M.: 1999, Solar Phys., 187, 59.

Schwartz, R. A., Csillaghy, A., Tolbert, A. K., Hurford, G. J., McTiernan, J., and Zarro, D.: 2002, Solar Phys., 210, 165.

Smith, D. M. et al.: 2002, Solar Phys., 210, 33.

Trottet, G., Rolli, E., Magun, A., Barat, C., Kuznetsov, A., Sunyaev, R., and Terekhov, O.: 2000, Astron. Astrophys., 356, 1067.

Uchida, Y.: 1996, Adv. Space Res. 17, 19.

Veronig, A., Steinegger, M., Otruba, W., Hanslmeier, A., Messerotti, M., Temmer, M., Gonzi, S., and Brunner, G.: 2000, Hvar. Obs. Bull., 24, No. 1, 195.

Wang, H., Qiu, J., Denker, C., Spirock, T., Chen, H., and Goode, P. R.: 2000, ApJ, 542, 1080 . 